\title{
Management of Airway in a Patient with Traumatic Subglottic Stenosis
}

\author{
Zia Arshad, Haider Abbas, Sulekha Saxena*, Jaishri Bogra
}

Department of Anaesthesia, KG’s MU, Lucknow, India.

Email: *dr.sulekha2008@rediffmail.com

Received January $20^{\text {th }}, 2013$; revised March $10^{\text {th }}, 2013$; accepted April $15^{\text {th }}, 2013$

Copyright (C) 2013 Zia Arshad et al. This is an open access article distributed under the Creative Commons Attribution License, which permits unrestricted use, distribution, and reproduction in any medium, provided the original work is properly cited.

\begin{abstract}
Tracheal stenosis or tracheal injury is a distressing condition. The silicone tracheal T-tube presents a substitute for stent of this complicated disease. We have come across a case of tracheal perforation in which tracheoplasty was planned. After traceoplasty silicone tracheal T-tube was placed as a stent. We were not able to connect silicone tracheal T-tube with Bain circuit with ET TUBE No. 8.5, 8, 7.5, 7 connector and it was difficult to ventilate the patient now. We have tried other ET TUBE connector and finally we succeeded to connect ET TUBE No. 5.5 connector with Bain circuit and we were able to ventilate the patient.
\end{abstract}

Keywords: Traceoplasty; Tracheal T-Tube; Subglottic Stenosis

\section{Introduction}

Asphyxia due to extrinsic tracheal compression is a distressing problem. We have come across a case of strangulation with tracheal perforation with the complaint of swelling and crepitation around neck and respiratory distress and inability to vocalize, and it was planned for tracheostomy.

\section{The Case Report}

A 40-year-old male patient was presented in Emergency Department of K G Medical University, Lucknow, India, after accidental strangulation, with the complaint of swelling and crepitation around neck and inability to vocalize. Patient was tracheostomised soon after the accident with No. 8 uncuffed tracheostomy tube at C7-T1 level. USG neck shows a communication between trachea and subcutaneous plane, i.e. tracheal perforation. CT neck was normal except the laryngeal edema. Bronchoscopy done, reveal bilateral vocal cord in paramedian position with subglottic stenosis.

Tracheoplasty was planned and patient was posted in Operation Theater for surgery. After securing an intravenous line, ASA monitors like pulseoximeter, ECG, NIBP were attached. Patient was premedicated with inj. Glycopyrrolate $0.2 \mathrm{mg}$, inj. Midazolam $2 \mathrm{mg}$ and inj.

\footnotetext{
"Corresponding author.
}

Fentanyl 100 microgram. Induction was done with inj. Propofol and uncuffed racheostomy tube was replaced with No. 7.5 cuffed endotracheal tube through the tracheostomy stoma. Anaesthesia was maintained with nitrous oxide, oxygen, isoflurane and vecuronium. 12 no silicone tracheal T-tube (Figure 1) was placed at C3 level and secured. We inspected the proximal and distal ends using the bronchoscope. Once we confirmed that the proximal end was below the vocal cords, the distal end was above the carina, and the airway was patent, the bronchoscope was withdrawn. At that time, muscle paralysis was withdrawn and patient was taken on propofol infusion with spontaneous respiration. When patient was generating adequate tidal volume, now we have tried to connect 12 no. tracheal tube with Bain circuit. When we have tried connector of 8.5, 8, 7.5 and 7 No. ETT tube, it was not fitted properly, now it was difficult to connect the 12 no. tracheal T-tube with Bain circuit and to ventilate the patient. Then we have tried connectors of other tubes and finally connector of 5.5 No. ETT tube was properly fitted with the 12 no. tracheal t-tube (Figure 2). Circuit was connected to 12 no. silicone tracheal T-tube through No. 5.5 ETT Connector. Nitrous and oxygen flow were increased to 10 and 6 liter/min respectively. Isoflurane was stopped. Tracheal stoma was closed (Figure 3). Patient was reversed with inj. Neostigmine. After reversal, tracheal T-tube opening was plugged. Now the patient was able to vocalize and breathing smoothly. 


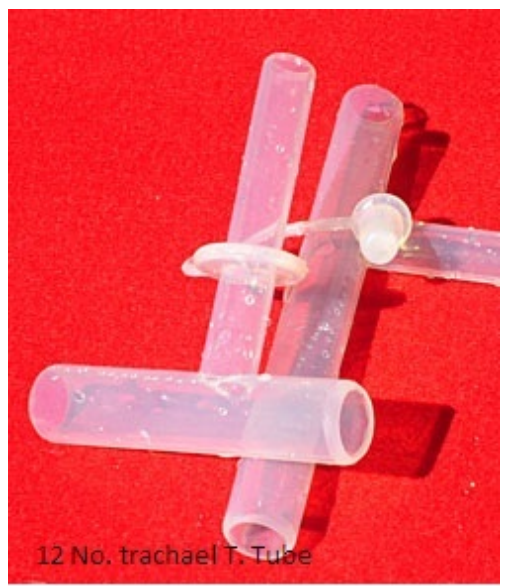

Figure 1. Silicone tracheal T-tube size No. 12.

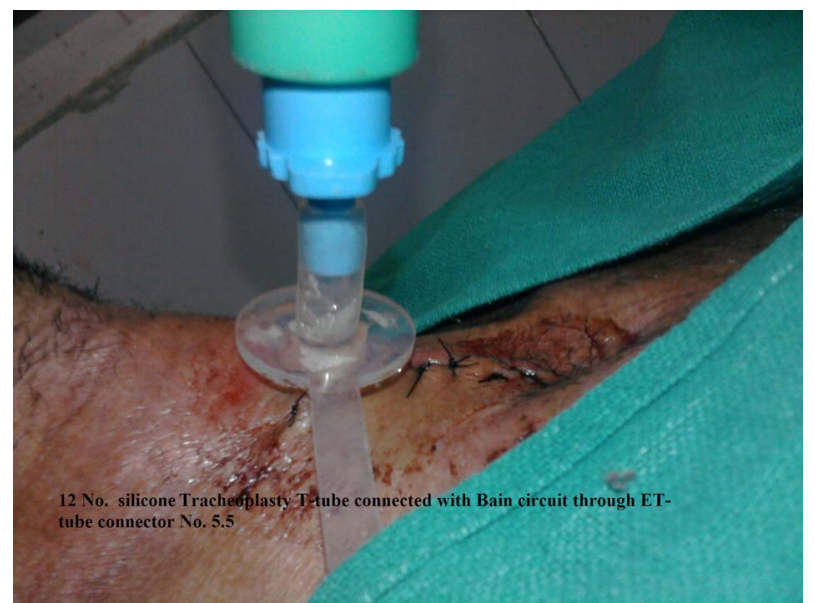

Figure 2. Tracheoplasty tube introduced and connected with Bain circuit with $5.5 \mathrm{~mm}$ endotracheal tube connector.

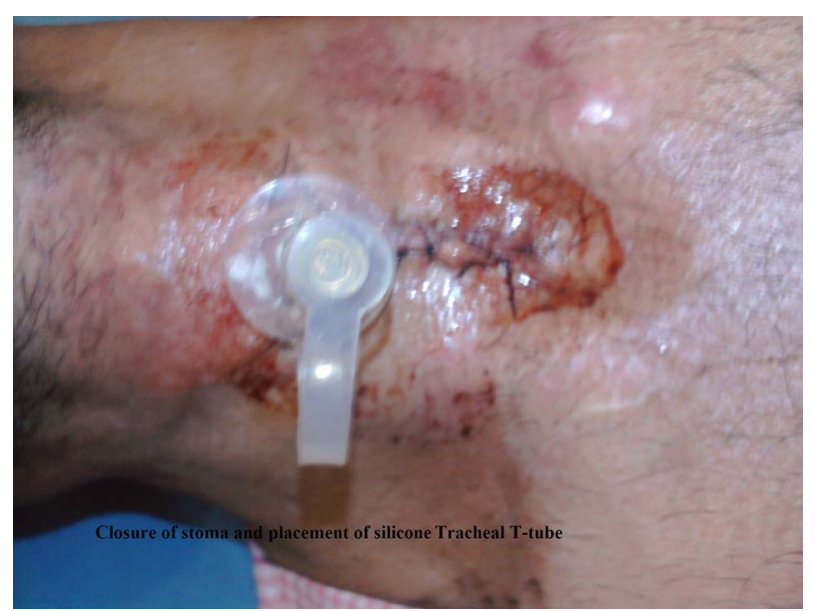

Figure 3. Closure of stoma with tacheoplasty tube in place.

\section{Discussion}

Anaesthetic management in airway surgeries is always a challenge to anaesthesiologist. Tactful management and situation based decisions are of immense importance along with the standard ASA guidelines for airway management. Montgomery [1], in 1965, reported the use of the T-tube tracheal stent to repair tracheal injuries. The tracheal $\mathrm{T}$ tube can maintain an adequate airway caliber and can act as a support for the tracheal wall to prevent collapse and can be used in the treatment of stenosis. T-tubes are a satisfactory alternative to tracheal resection [2] and are preferred over interbronchial stents for tracheal stenosis as T-tubes have decreased rate of migration, allow for frequent irrigation and suctioning, are easily removed in case of acute obstruction, and maintain a tracheostomy stoma [3,4]. T-tubes are well-tolerated and can be left in for years, if cared for properly $[5,6]$. A T-tube maintains a tracheostomy stoma, and a tracheostomy tube can easily be inserted in case of respiratory failure requiring ventilation. The treatment option chosen for this patient was a T-tube stent, and he elected to proceed with surgical insertion.

\section{REFERENCES}

[1] W. W. Montgomery, "Silicone Tracheal T-Tube," Annals of Otology, Vol. 83, 1974, pp. 71-75.

[2] C.-J. Huang, "Use of the Silicone T-Tube to Treat Tracheal Stenosis or Tracheal Injury," Annals of Thoracic and Cardiovascular Surgery, Vol. 7, No. 4, 2001, pp. 192-196.

[3] M. M. Wahidi and A. Ernst, "The Montgomery T-Tube Tracheal Stent," Clinics in Chest Medicine, Vol. 24, No. 3, 2003, pp. 437-443.

[4] W. W. Montgomery, "T-tube tracheal stent," Archives of Otolaryngology, Vol. 82, 1965, pp. 320-321.

[5] H. A. Gaissert, H. C. Grillo, D. J. Mathisen and J. C. Wain, "Temporary and Permanent Restoration of Airway Continuity with the Tracheal T-Tube," The Journal of Thoracic and Cardiovascular Surgery, Vol. 107, No. 2, 1994, pp. 600-606.

[6] J. R. Sonett, R. J. Keenan, P. F. Ferson, et al., "Endobronchial Management of Benign, Malignant, and Lung Transplantation Airway Stenoses," The Annals of Thoracic Surgery, Vol. 59, No. 6, 1995, pp. 1417-1422. doi:10.1016/0003-4975(95)00216-8 